ANNALES

POLONICI MATHEMATICI

LXI.1 (1995)

\title{
Factorization of uniformly holomorphic functions
}

\author{
by Luiza A. Moraes (Rio de Janeiro), Otilia W. Paques (Campinas) \\ and M. Carmelina F. Zaine (Campinas)
}

\begin{abstract}
Let $E$ be a complex Hausdorff locally convex space such that the strong dual $E^{\prime}$ of $E$ is sequentially complete, let $F$ be a closed linear subspace of $E$ and let $U$ be a uniformly open subset of $E$. We denote by $\Pi: E \rightarrow E / F$ the canonical quotient mapping. In $\S 1$ we study the factorization of uniformly holomorphic functions through $\pi$. In $\S 2$ we study $F$-quotients of uniform type and introduce the concept of envelope of $\mathrm{u} F$-holomorphy of a connected uniformly open subset $U$ of $E$. The main result states that the pull-back $\varepsilon_{\mathrm{u}}^{*}(U)$ of the envelope of uniform holomorphy of $\Pi(U)$ constructed by Paques and Zaine [9] is the envelope of $\mathrm{u} F$-holomorphy of $U$.
\end{abstract}

Introduction. We deal with the concept of uniform holomorphy (cf. [6]-[8]) of a holomorphic function $f: U \rightarrow \mathbb{C}$ in the case when $U$ is a nonvoid uniformly open subset of a complex Hausdorff locally convex space $E$. Let $F$ be a closed linear subspace of $E$, let $\Pi: E \rightarrow E / F$ be the canonical quotient mapping and let $I_{U}$ be the set of all continuous seminorms $\alpha$ on $E$ such that $U$ is open in $(E, \alpha)$. Let $H_{\mathrm{u}}(U)$ be the set of all uniformly holomorphic functions from $U$ into $\mathbb{C}$ and let $H_{\mathrm{u} F}(U)$ be the set of all $g \circ \Pi$ as $g$ ranges over $H_{\mathrm{u}}(\Pi(U))$. It is easy to show that $H_{\mathrm{u} F} \subset H_{\mathrm{u}}(U)$. In $\S 1$ we prove that if $U$ is a balanced uniformly open subset of $E$ and $F$ is a closed linear subspace of $(E, \alpha)$ for each $\alpha \in I_{U}$, then $g \circ \Pi$ is uniformly holomorphic if and only if $g$ is uniformly holomorphic.

The concepts of Riemann domain of uniform type and $F$-quotient of a Riemann domain were introduced in [9] and [4] respectively. Given a uniformly open subset $U$ of $E$ it is easy to verify that $\Pi(U)$ is a uniformly open subset of $E / F$ (cf. Ex. 3, §2). We have been unable to decide if an $F$-quotient of a Riemann domain of uniform type is always of uniform type. However, we give in $\S 2$ some non-trivial examples of $F$-quotients of a Riemann domain of uniform type which are of uniform type. In particular, we consider

1991 Mathematics Subject Classification: Primary 46G20.

Key words and phrases: uniformly holomorphic, envelope of holomorphy.

Research partially supported by CEPG-UFRJ, CNPq and FINEP. 
$\left(\varepsilon_{\mathrm{u}}(\Pi(U)), q_{\Pi}\right)$, the envelope of u-holomorphy of $\Pi(U)$ constructed in [9] and its pull-back $\left(\varepsilon_{\mathrm{u}}^{*}(U), \varphi^{*}\right)$. We prove that there exists an open mapping $\psi$ from $\varepsilon_{\mathrm{u}}^{*}(U)$ onto $\varepsilon_{\mathrm{u}}(\Pi(U))$ such that $\left(\varepsilon_{\mathrm{u}}(\Pi(U)), q_{\Pi}, \psi\right)$ is an $F$-quotient of uniform type of $\varepsilon_{\mathrm{u}}^{*}(U)$ satisfying the following: given $g \in H_{\mathrm{u}}(\Pi(U))$ there exists a uniform extension $\widetilde{f} \in H_{\mathrm{u}}\left(\varepsilon_{\mathrm{u}}^{*}(U)\right)$ of $f=g \circ \Pi$ which is defined by $\widetilde{g} \circ \psi$ where $\widetilde{g} \in H_{\mathrm{u}}\left(\varepsilon_{\mathrm{u}}(\Pi(U))\right)$ is a uniform extension of $g$. We also find that $\left(\varepsilon_{\mathrm{u}}^{*}(U), \varphi^{*}\right)$ is maximal in the sense of Definition 11.

We remark that the concept of envelope of $F$-holomorphy given in [4] of a connected open subset $U$ of a Banach space $E$ works also when $E$ is an arbitrary locally convex space. In particular, this paper extends the results of [4] to locally convex spaces with $H_{\mathrm{u}}(U)=H(U)$ and $H_{\mathrm{u}}(\Pi(U))=H(\pi(U))$. This is the case if $E$ is a dual of a separable Fréchet space endowed with the compact-open topology (cf. [5] and [8]).

Acknowledgements. The authors want to thank J. Ansemil and S. Ponte for many interesting discussions. Thanks are due also to the supporting agencies and to UFRJ and UNICAMP for many facilities.

Notation and terminology. Throughout this paper $E$ is a complex Hausdorff locally convex space whose strong dual $E^{\prime}$ is sequentially complete, $F$ is a closed linear subspace of $E$ and $\Pi: E \rightarrow E / F$ is the canonical quotient mapping. We refer to [2] for the terminology in infinite-dimensional complex analysis.

Let $\operatorname{cs}(E)$ be the set of all continuous seminorms on $E$. For each $\alpha \in$ $\operatorname{cs}(E)$, we denote by $(E, \alpha)$ the space $E$ endowed with the topology generated by $\alpha$, by $E_{\alpha}$ the normed space associated with $(E, \alpha)$, by $i_{\alpha}: E \rightarrow E_{\alpha}$ the canonical quotient mapping and by $B_{\alpha}(x, r)$ the open ball with center $x$ and radius $r$ in $(E, \alpha)$. Given an open subset $U$ of $E$ we write $i_{\alpha}(U)=U_{\alpha}$ and, as usual, $H(U)$ is the vector space of all holomorphic functions from $U$ into $\mathbb{C}$.

An open subset $U$ of $E$ is said to be uniformly open if there exists $\alpha \in \operatorname{cs}(E)$ such that $U$ is open in $(E, \alpha)$. Let $I_{U}$ denote the set of all such $\alpha \in \operatorname{cs}(E)$. We remark that $I_{U}$ is a directed subset of $\operatorname{cs}(E)$ that generates the topology of $E$.

If $U$ is a uniformly open subset of $E$, a holomorphic function $f: U \rightarrow \mathbb{C}$ is said to be uniformly holomorphic on $U$ if there exist $\alpha \in I_{U}$ and $f_{\alpha} \in H\left(U_{\alpha}\right)$ such that $f=f_{\alpha} \circ i_{\alpha}$. We denote by $H_{\mathrm{u}}(U)$ the vector space of all uniformly holomorphic functions from $U$ into $\mathbb{C}$.

The following well known result will be useful:

Proposition A. If $V$ is an open subset of a locally convex space $M$, $M_{S}$ is the associated Hausdorff space of $M, Q: M \rightarrow M_{S}$ is the canonical 
mapping and $V_{S}=Q(V)$, then $f \in H(V)$ if and only if there exists $f_{S} \in$ $H\left(V_{S}\right)$ such that $f=f_{S} \circ Q$.

The pair $(X, \varphi)$ is a Riemann domain over $E$ if $X$ is a nonvoid Hausdorff topological space and $\varphi: X \rightarrow E$ is a local homeomorphism. Instead of $(X, \varphi)$ we often write $X$. Given $A \subseteq X$, we write $A \sim \varphi(A)$ to indicate that $A$ is homeomorphic to $\varphi(A)$ under $\varphi / A$. A chart in $X$ is a connected open subset $V$ of $X$ such that $\varphi / V: V \rightarrow \varphi(V)$ is a homeomorphism. An atlas on $X$ is a collection $\left(V_{i}\right)_{i \in I}$ of charts which cover $X$. We recall that if $U$ is an open subset of $E$ and $i_{U}: U \rightarrow E$ is the inclusion mapping, then $\left(U, i_{U}\right)$ is a Riemann domain over $E$.

A Riemann domain $(X, \varphi)$ over $E$ is said to be a Riemann domain of uniform type (or, simply, a domain of uniform type) if there exists $\alpha \in \operatorname{cs}(E)$ such that for each $x \in X$, there is a neighborhood $V(x)$ of $x$ such that $V(x) \sim \varphi(V(x))$ and $\varphi(V(x))$ is open in $(E, \alpha)$. Let $I_{X}$ denote the set of all such $\alpha \in \operatorname{cs}(E)$. For every $\alpha \in I_{X}$ let $(X, \alpha)$ be the set $X$ endowed with the topology generated by the neighborhoods $V$ that satisfy the above definition. We denote by $X_{\alpha}$ the Hausdorff space associated with $(X, \alpha)$, i.e., $X_{\alpha}=(X, \alpha) / R$ where $R$ is the equivalence relation on $X$ defined by: $x R y$ if and only if $\alpha(\varphi(x)-\varphi(y))=0$ for all $x, y \in X$. For each $\alpha \in I_{X}$, let $I_{\alpha}: X \rightarrow X_{\alpha}$ be the canonical quotient mapping; it is clear that if we define $\varphi_{\alpha}: X_{\alpha} \rightarrow E_{\alpha}$ by $\varphi_{\alpha} \circ I_{\alpha}:=i_{\alpha} \circ \varphi$, then $\varphi_{\alpha}$ is a local homeomorphism and $\left(X_{\alpha}, \varphi_{\alpha}\right)$ is a Riemann domain over $E_{\alpha}$.

If $(X, \varphi)$ is a Riemann domain over $E$ and $(Y, \varrho)$ is a Riemann domain over a Hausdorff locally convex space $G$, a continuous mapping $f: X \rightarrow$ $Y$ is said to be holomorphic if there is an atlas $\left(V_{i}\right)_{i \in I}$ on $X$ such that $\varrho \circ f \circ\left(\varphi / V_{i}\right)^{-1}: \varphi\left(V_{i}\right) \rightarrow G$ is holomorphic for each $i \in I$. We shall denote by $H(X, Y)$ the class of all mappings $f: X \rightarrow Y$ which are holomorphic. When $Y=\mathbb{C}$ we write $H(X)$ instead of $H(X ; \mathbb{C})$.

If $(X, \varphi)$ is a domain of uniform type, and $G$ is a Hausdorff locally convex space, a holomorphic mapping $f: X \rightarrow G$ is said to be uniformly holomorphic if for each $\beta \in \operatorname{cs}(G)$ there exist $\alpha \in I_{X}$ and a holomorphic mapping $f_{\alpha} \in H\left(X_{\alpha}, G_{\beta}\right)$ such that $i_{\beta} \circ f=f_{\alpha} \circ I_{\alpha}$.

For other notations and basic results on uniform holomorphy we refer to [6] and [9].

1. Factorization of uniformly holomorphic mappings. Let $U$ be a uniformly open subset of $E$. For each $\alpha \in \operatorname{cs}(E)$ define $\bar{\alpha}(\bar{x}):=\inf \{\alpha(x+y)$ : $y \in F\}$ for $\bar{x}=\Pi(x) \in E / F$. It is well known that $\bar{\alpha} \in \operatorname{cs}(E / F)$ and the set $\left\{\bar{\alpha}: \alpha \in I_{U}\right\}$ generates the topology of $E / F$.

Proposition 1. Let $U$ be a uniformly open subset of $E$. Then:

(a) $\Pi(U)$ is uniformly open and $\bar{\alpha} \in I_{\Pi(U)}$ for every $\alpha \in I_{U}$. 
(b) If $g$ is a uniformly holomorphic function on $\Pi(U)$, then $f=g \circ \Pi$ is uniformly holomorphic on $U$.

Proof of (b). Let $g \in H_{\mathrm{u}}(\Pi(U))$. Since $\left\{\bar{\alpha}: \alpha \in I_{U}\right\}$ generates the topology of $E / F$, there exist $\alpha \in I_{U}$ and $g_{\bar{\alpha}} \in H\left(\Pi(U)_{\bar{\alpha}}\right)$ such that $g=g_{\bar{\alpha}} \circ i_{\bar{\alpha}}$ where $i_{\bar{\alpha}}: E / F \rightarrow(E / F)_{\bar{\alpha}}$ is the canonical quotient mapping and $\Pi(U)_{\bar{\alpha}}=i_{\bar{\alpha}}(\Pi(U))$. If $i: E \rightarrow(E, \alpha)$ is the identity mapping, $\Pi_{\alpha}$ : $(E, \alpha) \rightarrow(E / F, \bar{\alpha})$ is the quotient mapping and $k_{\bar{\alpha}}:(E / F, \bar{\alpha}) \rightarrow(E / F)_{\bar{\alpha}}$ is the canonical quotient mapping, it is clear that $k_{\bar{\alpha}} \circ \Pi_{\alpha} \circ i=i_{\bar{\alpha}} \circ \Pi$. Consequently, $f=g \circ \Pi=g_{\bar{\alpha}} \circ i_{\bar{\alpha}} \circ \Pi=g_{\bar{\alpha}} \circ k_{\bar{\alpha}} \circ \Pi_{\alpha} \circ i$ and so there exists $f_{\alpha}^{\prime}=g_{\bar{\alpha}} \circ k_{\bar{\alpha}} \circ \Pi_{\alpha} \in H(i(U))$ such that $f=f_{\alpha}^{\prime} \circ i$. By Proposition A, there exists $f_{\alpha} \in H\left(U_{\alpha}\right)$ so that $f_{\alpha}^{\prime}=f_{\alpha} \circ k_{\alpha}$ where $k_{\alpha}:(E, \alpha) \rightarrow E_{\alpha}$ is the canonical quotient mapping. So, $f=f_{\alpha}^{\prime} \circ i=f_{\alpha} \circ k_{\alpha} \circ i=f_{\alpha} \circ i_{\alpha}$ and we have $f \in H_{\mathrm{u}}(U)$.

The next result gives us a reciprocal for Proposition 1(b) when $F$ is a closed linear subspace of $(E, \alpha)$ for each $\alpha \in I_{U}$.

Proposition 2. Let $U$ be a balanced uniformly open subset of $E$ and let $F$ be a closed linear subspace of $(E, \alpha)$ for each $\alpha \in I_{U}$. If $f$ is uniformly holomorphic on $U$ and $f=g \circ \Pi$ for some $g \in H(\Pi(U))$, then $g$ is uniformly holomorphic on $\Pi(U)$.

Proof. We define $k_{\alpha}, i, \Pi_{\alpha}, k_{\bar{\alpha}}$ and $i_{\bar{\alpha}}$ as in the proof of Proposition 1. By hypothesis there exist $\alpha \in I_{U}$ and $f_{\alpha} \in H\left(U_{\alpha}\right)$ such that $f=f_{\alpha} \circ i_{\alpha}$. If $f_{\alpha}^{\prime} \in H(i(U))$ is defined by $f_{\alpha}^{\prime}=f_{\alpha} \circ k_{\alpha}$ it follows that $f=f_{\alpha} \circ i_{\alpha}=$ $f_{\alpha} \circ k_{\alpha} \circ i=f_{\alpha}^{\prime} \circ i$. By Theorem 2.3 of [1], $f=g \circ \Pi$ if and only if $d f(x) / F=0$ for all $x \in U$. Consequently, $0=d f(x)(y)=d f_{\alpha}^{\prime}(i(x))(i(y))$ for all $y \in F$, i.e., $d f_{\alpha}^{\prime}(i(x)) / F=0$ or $f_{\alpha}^{\prime}$ factors through $\Pi_{\alpha}(i(U))$. So there exists $g^{\prime} \in H\left(\Pi_{\alpha}(i(U))\right)$ such that $f_{\alpha}^{\prime}=g^{\prime} \circ \Pi_{\alpha}$. If $k: E / F \rightarrow(E / F, \bar{\alpha})$ is the identity mapping, then $g=g^{\prime} \circ k$ on $\Pi(U)$. Indeed, for every $x \in U$,

$$
g(\Pi(x))=f(x)=\left(f_{\alpha}^{\prime} \circ i\right)(x)=\left(g^{\prime} \circ \Pi_{\alpha} \circ i\right)(x)=\left(g^{\prime} \circ k\right)(\Pi(x)) .
$$

Since $k_{\bar{\alpha}} \circ \Pi_{\alpha} \circ i=i_{\bar{\alpha}} \circ \Pi, \Pi_{\alpha}(i(U)) \subseteq(E / F, \bar{\alpha})$ and $g^{\prime} \in H\left(\Pi_{\alpha}(i(U))\right.$, by Proposition A, there exists $g_{\bar{\alpha}}^{\prime} \in H\left(i_{\bar{\alpha}}(\Pi(U))\right)$ such that $g^{\prime}=g_{\bar{\alpha}}^{\prime} \circ k_{\bar{\alpha}}$ on $\Pi_{\alpha}(i(U))=k(\Pi(U))$ and it follows that for every $\Pi(x) \in \Pi(U)$,

$$
g(\Pi(x))=\left(g^{\prime} \circ k\right)(\Pi(x))=\left(g_{\bar{\alpha}}^{\prime} \circ k_{\bar{\alpha}} \circ k\right)(\Pi(x))=g_{\bar{\alpha}}^{\prime} \circ i_{\bar{\alpha}}(\Pi(x)) .
$$

So, there exist $\bar{\alpha} \in I_{\Pi(U)}$ and $g_{\bar{\alpha}}^{\prime} \in H\left(\Pi(U)_{\bar{\alpha}}\right)$ such that $g=g_{\bar{\alpha}}^{\prime} \circ i_{\bar{\alpha}}$ on $\Pi(U)$, i.e., $g \in H_{\mathrm{u}}(\Pi(U))$.

2. Uniformly holomorphic continuation. Let $(X, \varphi)$ be a Riemann domain over $E$. We say that $\left(X_{F}, \varphi_{F}, \psi\right)$ is an $F$-quotient of $X$ if $\left(X_{F}, \varphi_{F}\right)$ is a Riemann domain over $E / F$ and $\psi$ is a continuous open mapping from $X$ onto $X_{F}$ such that $\varphi_{F} \circ \psi=\Pi \circ \varphi$. The concept of $F$-quotient of a 
Riemann domain was introduced and studied in [4], where several examples are presented. Here we give some examples of Riemann domains of uniform type $X$ over $E$ which admit an $F$-quotient $\left(X_{F}, \varphi_{F}, \psi\right)$ such that $\left(X_{F}, \varphi_{F}\right)$ is also of uniform type. In this case we will say that $\left(X_{F}, \varphi_{F}, \psi\right)$ is an F-quotient of uniform type of $X$.

EXAMPLE 3. Let $U$ be a uniformly open subset of $E$, and $i_{U}: U \rightarrow E$ and $i_{\Pi}: \Pi(U) \rightarrow E / F$ the inclusion mappings. Then $\Pi(U)$ is a uniformly open subset of $E / F$ (cf. Proposition $1(\mathrm{a}))$ and it is clear that $\left(\Pi(U), i_{\Pi}, \Pi\right)$ is an $F$-quotient of $\left(U, i_{U}\right)$ which is of uniform type.

ExAmPle 4. Let $(X, \varphi)$ be a Riemann domain of uniform type over $E$, let $R$ be the equivalence relation defined on $X$ by $x R y$ if and only if $\varphi(x)-\varphi(y) \in F$ for $x, y \in X$ and denote by $X / R$ the quotient set by this equivalence, endowed with the quotient topology associated with the mapping $\psi$ from $X$ onto $X / R$ defined by $\psi(x):=\bar{x}$ (where $\bar{x}$ denotes the equivalence class of $x)$. We can define $\varphi_{F}: X / R \rightarrow E / F$ by $\varphi_{F}(\bar{x}):=\Pi(\varphi(x))$ for $\bar{x} \in X / R$ and it is easy to see that $\left(X / R, \varphi_{F}\right)$ is a Riemann domain over $E / F$. By hypothesis, there is $\alpha \in \operatorname{cs}(E)$ such that, for each $x \in X$, there exist a neighborhood $V(x)$ of $x$ and an $r>0$ satisfying $V(x) \sim \varphi(V(x))=B_{\alpha}(\varphi(x), r)$. Since $\varphi_{F} \circ \psi=\Pi \circ \varphi$, we have $\varphi_{F} \circ \psi(V(x))=\Pi \circ \varphi(V(x))=\Pi\left(B_{\alpha}(\varphi(x), r)\right)=B_{\bar{\alpha}}\left(\varphi_{F} \circ \psi(x), r\right)$. Since $\psi(V(x))$ is a neighborhood of $\psi(x)$ and $\varphi_{F}$ is injective on $\psi(V(x))$, it is clear that $\left(X / R, \varphi_{F}\right)$ is of uniform type and so $\left(X / R, \varphi_{F}, \psi\right)$ is an $F$-quotient of uniform type of $X$.

Let $(X, \varphi)$ and $(Y, \varrho)$ be two Riemann domains over $E$. A continuous mapping $j: X \rightarrow Y$ is said to be a morphism if $\varrho \circ j=\varphi$. The concept of envelope of uniform holomorphy of a Riemann domain of uniform type was introduced and studied in [9]. We recall that if $U$ is a connected uniformly open subset of $E$ and $\left(\varepsilon_{\mathrm{u}}(U), q\right)$ is constructed as in [9], the morphism $j^{\prime}: U \rightarrow$ $\varepsilon_{\mathrm{u}}(U)$ defined by $j^{\prime}(u):=\widehat{u}$, where $\widehat{u}(f):=f(u)$ for $f \in H_{\mathrm{u}}(U)$, is the envelope of uniform holomorphy of $U$. Analogously $\left(\varepsilon_{\mathrm{u}}(\Pi(U)), q_{\Pi}\right)$ is constructed and the morphism $j_{\Pi}: \Pi(U) \rightarrow \varepsilon_{\mathrm{u}}(\Pi(U))$ defined by $j_{\Pi}(\Pi(u)):=\widehat{\Pi(u)}$, where $\widehat{\Pi(u)}(g):=g(\Pi(u))$ for $g \in H_{\mathrm{u}}(\Pi(U))$, is the envelope of uniform holomorphy of $\Pi(U)$. Following the idea used in the proof of Propositions 6 and 7 and Corollary 8 of [4], we get a new construction of $\left(\varepsilon_{\mathrm{u}}(\Pi(U)), q_{\Pi}\right)$ and an open mapping $\psi: \varepsilon_{\mathrm{u}}(U) \rightarrow \varepsilon_{\mathrm{u}}(\Pi(U))$ such that $\psi\left(\varepsilon_{\mathrm{u}}(U)\right)$ is a connected topological subspace of $\varepsilon_{\mathrm{u}}(\Pi(U))$. We denote also by $q_{\Pi}$ the restriction of $q_{\Pi}$ to $\psi\left(\varepsilon_{\mathrm{u}}(U)\right)$.

EXAMPLE 5. By using the definition of the topology of $\varepsilon_{\mathrm{u}}(\Pi(U))$, it is easy to verify that $\left(\psi\left(\varepsilon_{\mathrm{u}}(U)\right), q_{\Pi}\right)$ is a Riemann domain of uniform type over $E / F$. So, $\left(\psi\left(\varepsilon_{\mathrm{u}}(U)\right), q_{\Pi}, \psi\right)$ is an $F$-quotient of $\left(\varepsilon_{\mathrm{u}}(U), q\right)$ of uniform type. 
The pull-back of $\left(\varepsilon_{\mathrm{u}}(\Pi(U)), q_{\Pi}\right)$ is, by definition, the Riemann domain $\left(\varepsilon_{\mathrm{u}}^{*}(U), \varphi^{*}\right)$ over $E$ where $\varepsilon_{\mathrm{u}}^{*}(U):=\left\{(H, a) \in \varepsilon_{\mathrm{u}}(\Pi(U)) \times E: q_{\Pi}(H)=\right.$ $\Pi(a)\}$ endowed with the topology induced on $\varepsilon_{\mathrm{u}}^{*}(U)$ by the product topology on $\varepsilon_{\mathrm{u}}(\Pi(U)) \times E$ and $\varphi^{*}(H, a):=a$ for $(H, a) \in \varepsilon_{\mathrm{u}}^{*}(U)$ (cf. [3] and [10]).

ExAmple 6 . Let $\psi: \varepsilon_{\mathrm{u}}^{*}(U) \rightarrow \varepsilon_{\mathrm{u}}(\Pi(U))$ be defined by $\psi(H, a):=H$ for $(H, a) \in \varepsilon_{\mathrm{u}}^{*}(U)$. We claim that the Riemann domain $\left(\varepsilon_{\mathrm{u}}^{*}(U), \varphi^{*}\right)$ is of uniform type and $\left(\varepsilon_{\mathrm{u}}(\Pi(u)), q_{\Pi}, \psi\right)$ is an $F$-quotient of uniform type of $\left(\varepsilon_{\mathrm{u}}^{*}(U), \varphi^{*}\right)$.

Let $(H, a) \in \varepsilon_{\mathrm{u}}^{*}(U)$. By hypothesis there exist $\bar{\alpha} \in \operatorname{cs}(E / F), r>0$ and a basic neighborhood $N_{\bar{\alpha}}(H, r)=\left\{H_{\bar{b}}: \bar{b} \in B_{\bar{\alpha}}(\overline{0}, r)\right\}$ of $H$ such that $N_{\bar{\alpha}}(H, r) \sim B_{\bar{\alpha}}\left(q_{\Pi}(H), r\right)$. We recall that $H_{\bar{b}}(g):=\sum(1 / n !) H\left(\widehat{d}_{\bar{b}}^{n} g\right)$ for all $g \in H_{\mathrm{u}}(\Pi(U))$ and $q_{\Pi}\left(H_{\bar{b}}\right)=q_{\Pi}(H)+\bar{b}$ (cf. [9]). Let

$$
V:=\left(N_{\bar{\alpha}}(H, r) \times B_{\alpha}(a, r)\right) \cap \varepsilon_{\mathrm{u}}^{*}(U) .
$$

It is clear that $V$ is a neighborhood of $(H, a)$.

We claim $\varphi^{*} / V$ is a homeomorphism between $V$ and $B_{\alpha}\left(\varphi^{*}(H, a), r\right)$. The continuity of $\varphi^{*} / V$ is clear. Let $\left(H_{\bar{b}_{1}}, c\right) \neq\left(H_{\bar{b}_{2}}, d\right)$ in $V$. If $c=d, H_{\bar{b}_{1}}$ must be different from $H_{\bar{b}_{2}}$ and consequently $\bar{b}_{1} \neq \bar{b}_{2}$ and $\Pi(c)=q_{\Pi}\left(H_{\bar{b}_{1}}\right)=$ $q_{\Pi}(H)+\bar{b}_{1} \neq q_{\Pi}(H)+\bar{b}_{2}=q_{\Pi}\left(H_{\bar{b}_{2}}\right)=\Pi(d)$, and we have a contradiction. So, we must have $c \neq d$ and it is clear that $\varphi^{*}\left(H_{\bar{b}_{1}}, c\right) \neq \varphi^{*}\left(H_{\bar{b}_{2}}, d\right)$. To prove that $\varphi^{*} / V$ is onto $B_{\alpha}(a, r)$ it is enough to show that for each $c \in B_{\alpha}(a, r)$ there exists $\bar{b} \in B_{\bar{\alpha}}(\overline{0}, r)$ satisfying $q_{\Pi}\left(H_{\bar{b}}\right)=\Pi(c)$. Take $\bar{b}=\Pi(c-a)$ and it is done. This completes the proof that $\left(\varepsilon_{\mathrm{u}}^{*}(U), \varphi^{*}\right)$ is a Riemann domain of uniform type.

Now, we show that $\left(\varepsilon_{\mathrm{u}}(\Pi(U)), q_{\Pi}, \psi\right)$ is an $F$-quotient of $\left(\varepsilon_{\mathrm{u}}^{*}(U), \varphi^{*}\right)$ of uniform type. It is clear from the definitions that $\psi$ is a continuous mapping from $\varepsilon_{\mathrm{u}}^{*}(U)$ onto $\varepsilon_{\mathrm{u}}(\Pi(U))$ such that $\Pi \circ \varphi^{*}=q_{\Pi} \circ \psi$. So, all we have to prove is that $\psi$ is open. It is enough to show that given any $(H, a) \in \varepsilon_{\mathrm{u}}^{*}(U)$, for every basic neighborhood $N_{\bar{\alpha}}(H, r)$ of $H$, we have

$$
\psi\left(\left[N_{\bar{\alpha}}(H, r) \times B_{\alpha}(a, r)\right] \cap \varepsilon_{\mathrm{u}}^{*}(U)\right)=N_{\bar{\alpha}}(H, r) .
$$

Let $K \in N_{\bar{\alpha}}(H, r)$, i.e., $K=H_{\bar{b}}$ for some $\bar{b} \in B_{\bar{\alpha}}(\overline{0}, r)$. Since $\Pi\left(B_{\alpha}(0, r)\right)=$ $B_{\bar{\alpha}}(\overline{0}, r)$, there exists $b_{1} \in B_{\alpha}(0, r)$ such that $\Pi\left(b_{1}\right)=\bar{b}$. It is clear that $\left(H_{\bar{b}}, a+b_{1}\right) \in N_{\bar{\alpha}}(H, r) \times B_{\alpha}(a, r)$ and since $q_{\Pi}\left(H_{\bar{b}}\right)=\Pi(a)+\bar{b}=\Pi\left(a+b_{1}\right)$ implies $\left(H_{\bar{b}}, a+b_{1}\right) \in \varepsilon_{\mathrm{u}}^{*}(U)$ we get

$$
N_{\bar{\alpha}}(H, r) \subseteq \psi\left(\left[N_{\bar{\alpha}}(H, r) \times B_{\alpha}(a, r)\right] \cap \varepsilon_{\mathrm{u}}^{*}(U)\right) .
$$

The other inclusion is trivial.

Let $\left(X_{F}, \varphi_{F}, \psi\right)$ be an $F$-quotient of uniform type of $X$ and take any $\bar{\alpha} \in I_{X_{F}}$. We denote by $X_{F \bar{\alpha}}$ the space $\left(X_{F}\right)_{\bar{\alpha}}$ and by $\varphi_{F \bar{\alpha}}$ the local homeomorphism $\varphi_{F \bar{\alpha}}: X_{F \bar{\alpha}} \rightarrow(E / F)_{\bar{\alpha}}$. If $I_{\bar{\alpha}}: X_{F} \rightarrow X_{F \bar{\alpha}}$ is the canonical 
quotient mapping, let $\bar{x}_{\bar{\alpha}}:=I_{\bar{\alpha}}(\bar{x})$ for all $\bar{x} \in X_{F}$, i.e., $\bar{x}_{\bar{\alpha}}=\left\{\bar{y} \in X_{F}\right.$ : $\left.\bar{\alpha}\left(\varphi_{F}(\bar{x})-\varphi_{F}(\bar{y})\right)=0\right\}$. We recall that $\varphi_{F \bar{\alpha}}\left(\bar{x}_{\bar{\alpha}}\right):=\varphi_{F}(\bar{x})+\bar{\alpha}^{-1}(\overline{0})$ for all $\bar{x} \in X_{F}$ and $\left(X_{F \bar{\alpha}}, \varphi_{F \bar{\alpha}}\right)$ is a Riemann domain over $(E / F)_{\bar{\alpha}}$.

LEMma 7. Let $U$ be a uniformly open subset of $E$. Suppose that $(X, \varphi)$ is a Riemann domain of uniform type over $E$ and $\left(X_{F}, \varphi_{F}, \psi\right)$ is an $F$-quotient of uniform type of $X$. Then:

(a) With every $\alpha \in I_{U}$ and $\bar{\beta} \in I_{X_{F}}$ we can associate $\gamma \in I_{U} \cap I_{X}$ such that $\bar{\gamma} \geq \bar{\alpha}, \bar{\gamma} \geq \bar{\beta}$, and so $\bar{\gamma} \in I_{X_{F}}$.

(b) Given $\bar{\gamma}, \bar{\beta} \in I_{X_{F}}$ so that $\bar{\beta} \leq \bar{\gamma}$, if $\widetilde{g}=\widetilde{g}_{\bar{\beta}} \circ I_{\bar{\beta}}$ for some $\widetilde{g}_{\bar{\beta}} \in H\left(X_{F \bar{\beta}}\right)$ then there exists $\widetilde{g}_{\bar{\gamma}} \in H\left(X_{F \bar{\gamma}}\right)$ satisfying $\widetilde{g}=\widetilde{g}_{\bar{\gamma}} \circ I_{\bar{\gamma}}$.

Proof. (a) Let $\alpha \in I_{U}$ and $\bar{\beta} \in I_{X_{F}}$. Since $X$ is of uniform type we can choose $\delta \in I_{X} \neq \emptyset$. As $\left\{\bar{\lambda}: \lambda \in I_{U}\right\}$ generates the topology of $E / F$ there exists $\alpha_{0} \in I_{U}$ such that $\bar{\beta} \leq \bar{\alpha}_{0}$. But since $I_{U}$ generates the topology of $E$ there exists $\gamma \in I_{U}$ such that $\delta, \alpha, \alpha_{0} \leq \gamma$. It is clear that $\bar{\gamma} \geq \bar{\alpha}, \bar{\beta}$, $\gamma \in I_{U} \cap I_{X}$ and $\bar{\gamma} \in I_{X_{F}}$.

(b) If we define $i_{\bar{\gamma} \bar{\beta}}:(E / F)_{\bar{\gamma}} \rightarrow(E / F)_{\bar{\beta}}$ by $i_{\bar{\gamma} \bar{\beta}} \circ i_{\bar{\gamma}}:=i_{\bar{\beta}}$, it is easy to verify that $i_{\bar{\gamma} \bar{\beta}}$ is a well defined continuous linear mapping from $(E / F)_{\bar{\gamma}}$ onto $(E / F)_{\bar{\beta}}$. Consequently, it is a holomorphic mapping. Analogously we define $I_{\bar{\gamma} \bar{\beta}}: X_{F \bar{\gamma}} \rightarrow X_{F \bar{\beta}}$ by $I_{\bar{\gamma} \bar{\beta}}\left(\bar{x}_{\bar{\gamma}}\right):=\bar{x}_{\bar{\beta}}$ for $\bar{x}_{\bar{\gamma}} \in X_{F \bar{\gamma}}$. As $\bar{\gamma}\left(\varphi_{F}(x)-\varphi_{F}(y)\right)=0$ implies $\bar{\beta}\left(\varphi_{F}(\bar{x})-\varphi_{F}(\bar{y})\right)=0$, it is easy to see that $I_{\bar{\gamma} \bar{\beta}}$ is well defined. It is also clear that $I_{\bar{\gamma} \bar{\beta}}$ is continuous and for every chart $V$ of $X_{F \bar{\gamma}}$ we have $i_{\bar{\gamma} \bar{\beta}}=\varphi_{F \bar{\beta}} \circ I_{\bar{\gamma} \bar{\beta}} \circ\left(\varphi_{F \bar{\gamma}} / V\right)^{-1}$. Consequently, $I_{\bar{\gamma} \bar{\beta}} \in H\left(X_{F \bar{\gamma}}, X_{F \bar{\beta}}\right)$. Now if $\widetilde{g}=\widetilde{g}_{\bar{\beta}} \circ I_{\bar{\beta}}$ with $\widetilde{g}_{\bar{\beta}} \in H\left(X_{F \bar{\beta}}\right)$ it is enough to define $\widetilde{g}_{\bar{\gamma}}: X_{F \bar{\gamma}} \rightarrow \mathbb{C}$ by $\widetilde{g}_{\bar{\gamma}}:=\widetilde{g}_{\bar{\beta}} \circ I_{\bar{\gamma} \bar{\beta}}$.

If $(Y, \varrho)$ is a Riemann domain of uniform type over $E / F$, then a morphism $j: \Pi(U) \rightarrow Y$ is said to be a uniform extension of $\Pi(U)$ if for each $g \in$ $H_{\mathrm{u}}(\Pi(U))$ there is a unique $\widetilde{g} \in H_{\mathrm{u}}(Y)$ such that $\widetilde{g} \circ j=g$. In this case $\widetilde{g}$ is said to be a uniform extension of $g$ to $Y$.

Definition 8. Let $(X, \varphi)$ be a Riemann domain of uniform type over $E$. A morphism $\gamma: U \rightarrow X$ is said to be a $u F$-extension of $U$ (uniform $F$-extension of $U)$ if there exist an $F$-quotient of uniform type $\left(X_{F}, \varphi_{F}, \psi\right)$ of $X$ and a morphism $\gamma_{\Pi}: \Pi(U) \rightarrow X_{F}$ such that:

(a) $\psi \circ \gamma=\gamma_{\Pi} \circ \Pi$.

(b) $\gamma_{\Pi}$ is a uniform extension of $\Pi(U)$.

Remark 9. In the above case, given $g \in H_{\mathrm{u}}(\Pi(U))$ there exists a uniform extension $\widetilde{f} \in H_{\mathrm{u}}(X)$ of $f=g \circ \Pi$ which is defined by $\widetilde{f}=\widetilde{g} \circ \psi$ where $\widetilde{g} \in H_{\mathrm{u}}\left(X_{F}\right)$ is a uniform extension of $g$. Indeed, since $g \in H_{\mathrm{u}}(\Pi(U))$, there exist $\alpha \in I_{U}$ and $g_{\bar{\alpha}} \in H\left(\Pi(U)_{\bar{\alpha}}\right)$ such that $g=g_{\bar{\alpha}} \circ i_{\bar{\alpha}}$ (where $i_{\bar{\alpha}}, \Pi(U)_{\bar{\alpha}}$ and $g_{\bar{\alpha}}$ are defined as in the proof of Proposition 1(b)). If $\widetilde{g} \in H_{\mathrm{u}}\left(X_{F}\right)$ is the 
uniform extension of $g$ to $X_{F}$ (whose existence is proved in [9]), then there exist $\bar{\beta} \in I_{X_{F}}$ and $\widetilde{g}_{\bar{\beta}} \in H\left(X_{F \bar{\beta}}\right)$ such that $\widetilde{g}=\widetilde{g}_{\bar{\beta}} \circ I_{\bar{\beta}}$. By Lemma 7 there exists $\gamma \in I_{U} \cap I_{X}$ so that $\bar{\gamma} \geq \bar{\alpha}$ and $\bar{\gamma} \geq \bar{\beta}$ and there exists $\widetilde{g}_{\bar{\gamma}} \in H\left(X_{F \bar{\gamma}}\right)$ satisfying $\widetilde{g}=\widetilde{g}_{\bar{\gamma}} \circ I_{\bar{\gamma}}$. Let $\widetilde{f}:=\widetilde{g} \circ \psi$. It is clear that $\widetilde{f}$ is continuous.

We claim that there exists a holomorphic mapping $\psi_{\bar{\gamma}}: X_{\gamma} \rightarrow X_{F \bar{\gamma}}$ such that $\psi_{\bar{\gamma}} \circ I_{\gamma}=I_{\bar{\gamma}} \circ \psi$ (recall that $I_{\gamma}: X \rightarrow X_{\gamma}$ is the canonical quotient mapping). If this is true, there exists $\widetilde{f}_{\gamma}:=\widetilde{g}_{\bar{\gamma}} \circ \psi_{\bar{\gamma}}$ such that $\widetilde{f}_{\gamma} \in H\left(X_{\gamma}\right)$ and $\widetilde{f}_{\gamma} \circ I_{\gamma}=\widetilde{g}_{\bar{\gamma}} \circ \psi_{\bar{\gamma}} \circ I_{\gamma}=\widetilde{g}_{\bar{\gamma}} \circ I_{\bar{\gamma}} \circ \psi=\widetilde{g} \circ \psi=\widetilde{f}$ and consequently $\widetilde{f} \in H_{\mathrm{u}}(X)$. So, it is clear that $\widetilde{f}=\widetilde{g} \circ \psi$ is a uniform extension of $f=g \circ \Pi$.

Now we are going to prove the claim. Let $\psi_{\bar{\gamma}}\left(I_{\gamma}(x)\right):=I_{\bar{\gamma}}(\psi(x))$ for $x \in X$. It is clear from the definition that $\psi_{\bar{\gamma}}\left(X_{\gamma}\right) \subseteq X_{F \bar{\gamma}}$. Given $x, y \in X$ such that $I_{\gamma}(x)=I_{\gamma}(y)$, we have $\gamma(\varphi(x)-\varphi(y))=0$. Let $\xi=\varphi(x)-\varphi(y) \in$ $\gamma^{-1}(0)$. From $\Pi \circ \varphi=\varphi_{F} \circ \psi$ and $\bar{\gamma}(\Pi(\xi))=0$ we get

$$
\bar{\gamma}\left[\varphi_{F}(\psi(x))-\varphi_{F}(\psi(y))\right]=\bar{\gamma}[\Pi(\varphi(x)-\varphi(y))]=\bar{\gamma}(\Pi(\xi))=0
$$

and so $I_{\bar{\gamma}}(\psi(x))=I_{\bar{\gamma}}(\psi(y))$, i.e., $\psi_{\bar{\gamma}}\left(I_{\gamma}(x)\right)=\psi_{\bar{\gamma}}\left(I_{\gamma}(y)\right)$. To prove the continuity of $\psi_{\bar{\gamma}}$ we take $x_{\gamma}=I_{\gamma}(x) \in X_{\gamma}$ and an arbitrary open neighborhood $V_{\bar{\gamma}}$ of $\psi_{\bar{\gamma}}\left(x_{\gamma}\right)$. We recall that $\bar{\gamma} \in I_{X_{F}}$. So there exist $r_{1}>0$ and an open neighborhood $V_{\bar{\gamma}}^{r_{1}}$ of $\psi_{\bar{\gamma}}\left(x_{\gamma}\right)$ such that $V_{\bar{\gamma}}^{r_{1}} \sim B_{\bar{\gamma}}\left(\varphi_{F \bar{\gamma}} \circ \psi_{\bar{\gamma}}\left(x_{\gamma}\right), r_{1}\right)$ and $V_{\bar{\gamma}}^{r_{1}} \subseteq V_{\bar{\gamma}}$; it is clear that for all $s \leq r_{1}$ there exists an open neighborhood $V_{\bar{\gamma}}^{s}$ of $\psi_{\bar{\gamma}}\left(x_{\gamma}\right)$ such that $V_{\bar{\gamma}}^{s} \subseteq V_{\bar{\gamma}}^{r_{1}}$ and $V_{\bar{\gamma}}^{s} \sim B_{\bar{\gamma}}\left(\varphi_{F \bar{\gamma}} \circ \psi_{\bar{\gamma}}\left(x_{\gamma}\right), s\right)$. Since $\psi(x) \in X_{F}$ there exist $r_{2}>0$ and an open neighborhood $U_{\bar{\gamma}}^{r_{2}}$ of $\psi(x)$ such that $U_{\bar{\gamma}}^{r_{2}} \sim B_{\bar{\gamma}}\left(\varphi_{F}(\psi(x)), r_{2}\right)$; for all $s \leq r_{2}$ there is an open neighborhood $U_{\bar{\gamma}}^{s}$ of $\psi(x)$ so that $U_{\bar{\gamma}}^{s} \subseteq U_{\bar{\gamma}}^{r_{2}}$ and $U_{\bar{\gamma}}^{s} \sim B_{\bar{\gamma}}\left(\varphi_{F}(\psi(x)), s\right)$. Let $r=\min \left\{r_{1}, r_{2}\right\}$. Then $U_{\bar{\gamma}}^{r}$ is the open neighborhood of $\psi(x)$ such that $\left.U_{\bar{\gamma}}^{r} \sim B_{\bar{\gamma}}\left(\varphi_{F}(\psi(x)), r\right)\right)$. Now,

$$
\begin{aligned}
i_{\bar{\gamma}}\left[B_{\bar{\gamma}}\left(\varphi_{F}(\psi(x)), r\right)\right] & =B_{\bar{\gamma}}\left(i_{\bar{\gamma}} \circ \varphi_{F}(\psi(x)), r\right)=B_{\bar{\gamma}}\left(\varphi_{F \bar{\gamma}}\left(I_{\bar{\gamma}} \circ \psi(x)\right), r\right) \\
& =B_{\bar{\gamma}}\left(\varphi_{F \bar{\gamma}}\left(\psi_{\bar{\gamma}}\left(x_{\gamma}\right)\right), r\right)
\end{aligned}
$$

implies $I_{\bar{\gamma}}\left(U_{\bar{\gamma}}^{r}\right)=V_{\bar{\gamma}}^{r}$. On the other hand, given $B_{\gamma}(\varphi(x), r)$ it is clear that $\Pi\left(B_{\gamma}(\varphi(x), r)\right)=B_{\bar{\gamma}}(\Pi(\varphi(x)), r)=B_{\bar{\gamma}}\left(\varphi_{F}(\psi(x)), r\right)$. Since $\gamma \in I_{X}$ there are $r_{0} \leq r$ and an open neighborhood $W$ of $x$ in $X$ such that $\varphi(W) \sim$ $B_{\gamma}\left(\varphi(x), r_{0}\right)$. As $\Pi \circ \varphi=\varphi_{F} \circ \psi$ it follows that

$$
\begin{aligned}
\psi(W) & =\left(\varphi_{F} / U_{\bar{\gamma}}^{r}\right)^{-1} \circ \Pi \circ \varphi(W)=\left(\varphi_{F} / U_{\bar{\gamma}}^{r}\right)^{-1}\left(B_{\bar{\gamma}}\left(\varphi_{F}(\psi(x)), r_{0}\right)\right) \\
& \subseteq\left(\varphi_{F} / U_{\bar{\gamma}}^{r}\right)^{-1}\left(B_{\bar{\gamma}}\left(\varphi_{F}(\psi(x)), r\right)\right)=U_{\bar{\gamma}}^{r} .
\end{aligned}
$$

So $I_{\gamma}(W)$ is an open subset of $X_{\gamma}$ containing $I_{\gamma}(x)$ such that $\psi_{\bar{\gamma}}\left(I_{\gamma}(W)\right)=$ $I_{\bar{\gamma}}(\psi(W)) \subseteq V_{\bar{\gamma}}^{r} \subseteq V_{\bar{\gamma}}$ and we have the continuity of $\psi_{\bar{\gamma}}$.

Finally, $\psi_{\bar{\gamma}}$ is holomorphic if there exists a holomorphic mapping $\Pi_{\bar{\gamma}}$ : $E_{\gamma} \rightarrow(E / F)_{\bar{\gamma}}$ satisfying $\Pi_{\bar{\gamma}}=\varphi_{F \bar{\gamma}} \circ \psi_{\bar{\gamma}} \circ\left(\varphi_{\gamma} / V\right)^{-1}$ for every chart $V$ of $X_{\gamma}$. Define $\Pi_{\bar{\gamma}}\left(i_{\gamma}(x)\right):=i_{\bar{\gamma}}(\Pi(x)) \in(E / F)_{\bar{\gamma}}$ for $x \in X$. It is clear that 
$\Pi_{\bar{\gamma}}$ is a well defined mapping from $E_{\gamma}$ onto $(E / F)_{\bar{\gamma}}$. The linearity of $\Pi_{\bar{\gamma}}$ follows from the linearity of $i_{\gamma}, i_{\bar{\gamma}}$ and $\Pi$. Now, for all $i_{\gamma}(x) \in E_{\gamma}$ we have $\bar{\gamma}\left[\Pi_{\bar{\gamma}}\left(i_{\gamma}(x)\right)\right]=\bar{\gamma}\left[i_{\bar{\gamma}}(\Pi(x))\right]=\bar{\gamma}(\Pi(x)) \leq \gamma(x)=\gamma\left(i_{\gamma}(x)\right)$ and consequently $\Pi_{\bar{\gamma}}$ is continuous. (We remark that we denote by $\gamma$ the norm in $E_{\gamma}$ associated with $\gamma$ since $\inf \left\{\gamma(x+y): y \in \gamma^{-1}(0)\right\}=\gamma(x)$; analogously for $\bar{\gamma}$.) Since $\Pi_{\bar{\gamma}}$ is a continuous linear mapping, it is holomorphic. It is easy to verify that $\Pi_{\bar{\gamma}} \circ \varphi_{\gamma}=\varphi_{F \bar{\gamma}} \circ \psi_{\bar{\gamma}}$ and this completes the proof.

EXAmPLE 10. The morphism $j^{\prime}: U \rightarrow \varepsilon_{\mathrm{u}}(U)$ defined by $j^{\prime}(u):=\widehat{u}$ is a $u F$-extension of $U$. Indeed, in Example 5 we define $\left(\psi\left(\varepsilon_{\mathrm{u}}(U)\right), q_{\Pi}, \psi\right)$ and prove that it is an $F$-quotient of uniform type of $\left(\varepsilon_{\mathbf{u}}(U), q\right)$ such that $\psi\left(\varepsilon_{\mathrm{u}}(U)\right) \subseteq \varepsilon_{\mathrm{u}}(\Pi(U))$. Since $j_{\Pi}: \Pi(U) \rightarrow \varepsilon_{\mathrm{u}}(\Pi(U))$ is a uniform extension of $\Pi(U)$ such that $j_{\Pi}(\Pi(U)) \subseteq \psi\left(\varepsilon_{\mathrm{u}}(U)\right)$, it is easy to show that $j_{\Pi}$ is a $\mathrm{u} F$ extension of $\Pi(U)$. From the definitions it is also clear that $\psi \circ j^{\prime}=j_{\Pi} \circ \Pi$.

Definition 11. Let $(X, \varphi)$ be a Riemann domain of uniform type over $E$. A morphism $\gamma: U \rightarrow X$ is said to be an envelope of $\mathrm{u} F$-holomorphy of $U$ if:

(a) $\gamma$ is a $u F$-extension of $U$.

(b) If $\mu: U \rightarrow Z$ is a $u F$-extension of $U$, then there is a morphism $\nu: Z \rightarrow X$ such that $\nu \circ \mu=\gamma$.

It is clear that if $\gamma: U \rightarrow X$ and $\gamma^{\prime}: U \rightarrow X^{\prime}$ are two envelopes of $\mathrm{u} F$-holomorphy of $U$ then the Riemann domains $X$ and $X^{\prime}$ are isomorphic. In other words, the envelope of $u F$-holomorphy of $U$, if it exists, is unique up to isomorphism.

THEOREM 12. Let $U$ be a connected uniformly open subset of $E$ and let $\left(\varepsilon_{\mathrm{u}}^{*}(U), \varphi^{*}\right)$ be the pull-back of $\left(\varepsilon_{\mathrm{u}}(\Pi(U)), q_{\Pi}\right)$. Then the mapping $\gamma$ : $U \rightarrow \varepsilon_{\mathrm{u}}^{*}(U)$ defined by $\gamma(u):=(\widehat{\Pi(u)}, u)$ for $u \in U$ is an envelope of $\mathrm{u} F$ holomorphy of $U$.

Proof. It is clear that $\varphi^{*} \circ \gamma=i_{U}$ where $i_{U}: U \rightarrow E$ is the inclusion. So, $\gamma$ is a morphism if it is continuous. Given $u \in U$, take a neighborhood of $(\widehat{\Pi(u)}, u)$ in $\varepsilon_{\mathrm{u}}^{*}(U)$ of the form $(V \times W) \cap \varepsilon_{\mathrm{u}}^{*}(U)$ where $V$ is a neighborhood of $\widehat{\Pi(u)}$ in $\varepsilon_{\mathrm{u}}(\Pi(U))$ and $W$ is a neighborhood of $u$ in $E$. Without loss of generality, we can suppose $W \subset U$. Since $j_{\Pi}: \Pi(U) \rightarrow \varepsilon_{\mathbf{u}}(\Pi(U))$ is an extension of $\Pi(U)$ there is an open set $V_{1} \subset \Pi(U)$ such that $\Pi(u) \in V_{1}$ and $j_{\Pi}\left(V_{1}\right) \subseteq V$. Let $V_{2}:=W \cap \Pi^{-1}\left(V_{1}\right)$. It is clear that for every $a \in V_{2}$ we have $\gamma(a) \in(V \times W) \cap \varepsilon_{\mathrm{u}}^{*}(U)$ and this gives the continuity of $\gamma$ in $u$. From Example $6,\left(\varepsilon_{\mathrm{u}}^{*}(U), \varphi^{*}\right)$ is a Riemann domain of uniform type over $E$ and $\left(\varepsilon_{\mathrm{u}}(\Pi(U)), q_{\Pi}, \psi\right)$ is an $F$-quotient of uniform type of $\left(\varepsilon_{\mathrm{u}}^{*}(U), \varphi^{*}\right)$. Since $j_{\Pi}: \Pi(U) \rightarrow \varepsilon_{\mathrm{u}}(\Pi(U))$ is a uniform extension of $\Pi(U)$ (cf. [9]) and clearly $\psi \circ \gamma=j_{\Pi} \circ \Pi$, it follows that $\left(\varepsilon_{\mathrm{u}}^{*}(U), \varphi^{*}\right)$ is a $\mathrm{u} F$-extension of $U$. 
Now, if $(Z, \varrho)$ is a Riemann domain of uniform type over $E$ and $\mu: U \rightarrow Z$ is a $\mathrm{u} F$-extension of $U$ there are an $F$-quotient of uniform type $\left(Z_{F}, \varrho_{F}, \psi_{F}\right)$ of $Z$ and $\mu_{\Pi}: \Pi(U) \rightarrow Z_{F}$ such that $\psi_{F} \circ \mu=\mu_{\Pi} \circ \Pi$ and $\mu_{\Pi}$ is a uniform extension of $\Pi(U)$. From the maximality of $\varepsilon_{\mathrm{u}}(\Pi(U))$ (cf. [9]) there is a morphism $\mu_{F}: Z_{F} \rightarrow \varepsilon_{\mathrm{u}}(\Pi(U))$ such that $\mu_{F} \circ \mu_{\Pi}=j_{\Pi}$. We define $\nu: Z \rightarrow$ $\varepsilon_{\mathrm{u}}^{*}(U)$ by $\nu(z):=\left(\left(\mu_{F} \circ \psi_{F}\right)(z), \varrho(z)\right)$. Since $\left(q_{\Pi} \circ \mu_{F} \circ \psi_{F}\right)(z)=\Pi \circ \varrho(z)$, we have $\nu(z) \in \varepsilon_{\mathrm{u}}^{*}(U)$ for every $z \in Z$. It is easy to verify that $\nu$ is a morphism and $\nu \circ \mu=\gamma$.

Rem a r k 13. We have the following generalization: Let $G$ be a complete Hausdorff locally convex space and $f \in H_{\mathrm{u}}(U, G)$ such that $f=g \circ \Pi$ where $g \in H_{\mathrm{u}}(\Pi(U), G)$. From Theorem 2.5 of [9], there exists a uniform extension $\widetilde{g}: \varepsilon_{\mathrm{u}}(\Pi(U)) \rightarrow G$ of $g$. If $\widetilde{f}:=\widetilde{g} \circ \psi$, where $\psi: \varepsilon_{\mathrm{u}}^{*}(U) \rightarrow \varepsilon_{\mathrm{u}}(\Pi(U))$ is defined as in Example 6, then a small change in the argument used in Remark 9 shows that $\widetilde{f}$ is a uniform extension of $f$.

Finally, we establish the relation between $\varepsilon_{\mathrm{u}}^{*}(U)$ and $\varepsilon_{\mathrm{u}}(U)$.

Remark 14. There exists a morphism $\delta: \varepsilon_{\mathrm{u}}(U) \rightarrow \varepsilon_{\mathrm{u}}^{*}(U)$ satisfying $\delta \circ j^{\prime}=\gamma$ (where $\gamma$ is defined in Theorem 12 and $j^{\prime}$ in Example 10).

Pr o of. From Example 10 we know that $j^{\prime}$ is a $u F$-extension of $U$. Since, by Theorem 12, $\gamma: U \rightarrow \varepsilon_{\mathrm{u}}^{*}(U)$ is an envelope of $u F$-holomorphy of $U$, the existence of such $\delta$ follows from the maximality of $\varepsilon_{\mathrm{u}}^{*}(U)$.

\section{References}

[1] R. Aron, L. Moraes and R. Ryan, Factorization of holomorphic mappings in infinite dimensions, Math. Ann. 277 (1987), 617-628.

[2] S. Dineen, Complex Analysis in Locally Convex Spaces, North-Holland Math. Stud. 57, North-Holland, Amsterdam, 1981.

[3] P. Hilton, Tópicos de Álgebra Homológica, 8o Colóquio Brasileiro de Matemática, IME-Universidade de São Paulo, Brasil, 1971.

[4] L. Moraes, O. W. Paques and M. C. F. Zaine, F-quotients and envelope of F-holomorphy, J. Math. Anal. Appl. 163 (2) (1992), 393-405.

[5] J. Mujica, Domain of holomorphy in (DFC)-spaces, in: Functional Analysis, Holomorphy and Approximation Theory, Lecture Notes in Math. 843, Springer, Berlin, 1980, 500-533.

[6] L. Nach bin, Uniformité d'holomorphie et type exponentiel, in: Séminaire P. Lelong 1970, Lectures Notes in Math. 205, Springer, Berlin, 1971, 216-224.

[7] -, Recent developments in infinite dimensional holomorphy, Bull. Amer. Math. Soc. 79 (1973), 625-640.

[8] -, On pure uniform holomorphy in spaces of holomorphic germs, Results in Math. 8 (1985), 117-122.

[9] O. W. Paques and M. C. Zaine, Uniformly holomorphic continuation, J. Math. Anal. Appl. 123 (2) (1987), 448-454. 
[10] M. Schottenloher, The Levi problem for domains spread over locally convex spaces with a finite dimensional Schauder decomposition, Ann. Inst. Fourier (Grenoble) 26 (4) (1976), 207-237.

Luiza A. Moraes

INSTITUTO DE MATEMÁTICA

UNIVERSIDADE FEDERAL DO RIO DE JANEIRO

C.P. 68530

CEP 21945-970 RIO DE JANEIRO, RJ, BRASIL
Otilia W. Paques

M. Carmelina F. Zaine DEPARTAMENTO DE MATEMÁTICA INSTITUTO DE MATEMÁTICA ESTATÍSTICA E CIÊNCIA DA COMPUTAÇÃO UNIVERSIDADE ESTADUAL DE CAMPINAS C.P. 6065 , CEP 13081-970 CAMPINAS, SP, BRASIL

Reçu par la Rédaction le 13.4.1992

Révisé le 11.4.1994 